\title{
The protective effect of ginko bilboa leaves injection on the brain dopamine in the rat model of cerebral ischemia/reperfusion injury
}

\author{
Jin $\mathrm{Gu}^{1}$, Xin-Rong $\mathrm{He}^{1}$, Li-Ping Chen ${ }^{2}$
}

1. Traditional Chinese Medicine Pharmacy of PLA General Hospital, Beijing 100853, China

2. Department of traditional chinese medicine of PLA General Hospital, Beijing 100853, China

\begin{abstract}
:
Background: Ginkgo Bilboa injection has had been clinically applied to restore the damaged cells and tissues due to the ischemia through improving the cerebral blood supply and decreasing the oxygen consumption.

ObjectiveAim: To evaluate the Ginkgo Bilboa injection's therapeutic role towards ischemia/ reperfusion (I/R) injury through determination of monoamine neurotransmitter dopamine (DA) in corpus striatum.

Methods: After the incomplete global cerebral ischemia and reperfusion models were prepared, rats were randomly assigned into four groups: sham-operated group, ischemia-reperfusion group, nimodipine injection group, and Ginkgo Biloba injection group. The cerebrospinal fluid in the rat brain striatum at different time points was collected with microdialysis, and the level of monoamine neurotransmitters dopamineDA was determined by high performance liquid chromatography (HPLC) with electrochemical detector (ECD).

Results: The dopamineDA content in cerebral ischemia model group was significantly higher than that in the sham-operated group $(\mathrm{P}<0.05)$ at the $30 \mathrm{~min}$. However, the $\mathrm{DA}$ level in nimodipine injection group and Ginkgo Biloba injection group were lower than the model group $(\mathrm{P}<0.05)$. The dopamineDA level in Ginkgo Biloba injection group gradually decreased, and was significantly different from the model group $(\mathrm{P}<0.05)$.

Conclusion: Ginkgo Biloba injection can could significantly inhibit brain I/R injury, as demonstrated by prevention of excessive release of dopamineDA in striatum.
\end{abstract}

Key Words: MD-HPLC-ECD; Ginkgo Biloba injection; ischemia/reperfusion; dopamineDA

DOI: http://dx.doi.org/10.4314/ahs.v14i3.31

\section{Introduction}

Ischemic cerebrovascular disease occurs occured when the blood vessel is was blocked, and it is was often resulted from a clot formed from fat and cholesterol. During ischemic cerebrovascular disease, the brain cells suffered from the lack of nutrients and oxygen due to the difficulty of blood to reach brain. Ischemic cerebrovascular disease has had been regarded as one of the most severe diseases that lead to death 1 . After the restoration of blood flow in the patients with cerebral stroke, cerebral ischemia-reperfusion (I/R) injury often occursoccurred, resulting in more severe neurological deficits2. I/ $\mathrm{R}$ injury has had been closely related with

\footnotetext{
* Corresponding author:

Jin $\mathrm{Gu}$,

Traditional Chinese Medicine Pharmacy of

PLA General Hospital,

28\# Fuxing road Beijing, 100853, China

E-mail: GuJin20130331@163.com
}

imbalance of ions, free radicals, and inflammatory responses 3 .

Dopamine (DA) is was an important monoamine neurotransmitter and hormone, and has had been demonstrated to play various important physiological roles 4. Cerebral ischemia can could induce the release of dopamineDA in corpus striatum, and the pre-depletion of dopamineDA in corpus striatum can could protect $\mathrm{I} / \mathrm{R}$ injury. Additionally, some studies have had found that dopamineDA plays played a key role in regulating the neurogenesis 5 . The monitoring of dopamineDA level in corpus striatum can could well reflect the extent of injury, and will would be helpful for understanding the therapeutic role of drugs towards I/R injury.

The injection containing Ginkgo Bilboa extract has had been demonstrated to exhibit multiple pharmacological activities, including anti-oxidant, elevation of blood flow. The present study aims aimed to evaluate the influence of Ginkgo Bilboa injection towards the levels of dopamineDA in corpus striatum of rats with $I / R$ injury using the combination methods of microdialysis (MD) and high performance liquid chromatography 
(HPLC).

\section{Materials and Methods:}

\section{Chemicals and Reagents:}

Ginkgo Bilboa injection (batch number: E7161) was purchased from Zhonghao international company. Nimodipine injection (batch number: BXFH2D3) was obtained from Bayer company. DopamineDA was obtained from national institutes for food and drug control.

\section{Animals and treatments:}

Male SD rats (170-210 g) were purchased from General Hospital of the Chinese People's Liberation Army. The animals were cared for according to the Animal Facility Guidelines of General Hospital of the Chinese People's Liberation Army.

The rats were divided into 4 groups, and each group included 6 rats. One group was sham-operated group. One group was ischemia-reperfusion model in which the animals were given ischemic preconditioning followed the ischemia-reperfusion injury procedure (portal triad clamping for $30 \mathrm{~min}$, and blood flow restoration for $30 \mathrm{~min})$. One group was nimodipine injection group in which $4.5 \mathrm{mlmL} / \mathrm{kg}$ injection (i.p.) was given before the establishment of model. The final group was Ginkgo Bilboa injection group in which $0.9 \mathrm{mlmL} / \mathrm{kg}$ injection (i.p.) was given before the establishment of model. All the rats received intraperitoneal injection once. All experiments were completed in the animal experimental center of People's Liberation Army General Hospital. Experiments were conducted in accordance with animal ethical requirements.

\section{Microdialysis sampling}

All collections were made in a Raturn (BASi, West Lafayette, IN) while the animal kept awake and acitve freely. Collections probes were acutely implanted after surgery. Using a syringe pump (BASi BEE), a $30 \mathrm{~min}$ flush was performed through the microdialysis probe at $1 \mu \mathrm{L} / \mathrm{min}$ after which collections began at $1 \mu \mathrm{L} / \mathrm{min}$ for $60 \mathrm{~min}$ for 5 hours. Samples were stored on ice, and kept frozen at $-80 \mathrm{oC}$ until analysis.

The analysis of dopamine (DA) using HPLC-ECD method

The separation of dopamine (DA) was performed using HPLC. The Eclipse XDB-C18 column $(250 \mathrm{~mm} \times 4.6$ $\mathrm{mm}, 5 \mu \mathrm{m})$ was used, and the mobile phase contained $85 \mathrm{mM}$ citric acid, $100 \mathrm{mM}$ sodium acetate, $0.2 \mathrm{mM}$ Na2-EDTA, $0.9 \mathrm{mM}$ octane sulfonic natrium, $300 \mathrm{~mL}$ methanol ( $\mathrm{pH}=3.7)$. The elute velocity was $1 \mathrm{ml} / \mathrm{min}$, and the column temperature was maintained at $35 \mathrm{oC}$. The applied potentials $(\mathrm{mV})$ : conditioning cell $=+10 \mathrm{~V}$; analytical cell, $\mathrm{E} 1=+50 \mathrm{~V} ; \mathrm{E} 2=+340 \mathrm{~V}$.

Statistical analysis

The data was given as mean \pm S.D. Statistical differences were evaluated using the two-tailed Student's t-test and considered significant at the ${ }^{*} \mathrm{p}<0.05$ level.

\section{Results:}

\section{Method development:}

$20 \mu \mathrm{L}$ dopamineDA was injected and analyzed using several concentrations $(3.2 \mathrm{ng} / \mathrm{mL}, 8 \mathrm{ng} / \mathrm{mL}, 16 \mathrm{ng} /$ $\mathrm{mL}, 40 \mathrm{ng} / \mathrm{mL}$, and $80 \mathrm{ng} / \mathrm{mL}$ ). The representative chromatography figure was given in Fig. 1. The linear regression equation was $\mathrm{Y}=80.2080 \mathrm{X}-5.4924$, in which $\mathrm{Y}$ represented the peak area, and $\mathrm{X}$ represented the

Table 1 The alteration of DA concentrations in rat brain (mean \pm S.D., $\mathrm{n}=6$ ), unit: $\mathrm{ng} / \mathrm{mL}$

\begin{tabular}{|c|c|c|c|c|c|c|c|c|c|c|c|}
\hline \multirow[t]{4}{*}{ Jups } & \multicolumn{11}{|c|}{ Time/min } \\
\hline & \multirow[t]{3}{*}{20} & \multirow[t]{3}{*}{40} & 60 & 90 & 120 & 140 & 160 & 180 & 200 & 220 & 240 \\
\hline & & & (i.p. & (ischemiamode & (reperfusion & & & & & & \\
\hline & & & administration) & 1 begin $)$ & begin） & & & & & & \\
\hline & $27.4 \pm 7.9$ & $34.6 \pm 27.8$ & $25.5 \pm 11.6$ & $22.3 \pm 12.7$ & $20.3 \pm 5.7^{* *}$ & $29.5 \pm 13.4$ & $28.6 \pm 15.3$ & $19.7 \pm 6.1^{*}$ & $31.8 \pm 11.1$ & $31.9 \pm 15.8$ & $18.7 \pm 8.9$ \\
\hline & $25.3 \pm 1.6$ & $29.0 \pm 1.8$ & $23.9 \pm 3.6$ & $24.9 \pm 3.4$ & $95.7 \pm 0.2$ & $23.6 \pm 10.2$ & $21.4 \pm 6.1$ & $43.0 \pm 20.3$ & $27.4 \pm 17.3$ & $22.9 \pm 13.6$ & $17.2 \pm 11.8$ \\
\hline & $28.0 \pm 2.3$ & $32.3 \pm 20.9$ & $27.0 \pm 4.9$ & $38.2 \pm 18.2$ & $32.5 \pm 24.3^{* *}$ & $39.7 \pm 29.0$ & $49.8 \pm 18.4^{7}$ & $40.5 \pm 13.2^{\#}$ & $23.9 \pm 16.2$ & $24.3 \pm 14.4$ & $17.6 \pm 12.9$ \\
\hline & $24.4 \pm 4.9$ & $32.8 \pm 5.1$ & $28.3 \pm 7.3$ & $31.2 \pm 6.0$ & $54.5 \pm 22.7^{*, \#}$ & $40.5 \pm 7.2^{*}$ & $32.3 \pm 7.8$ & $30.7 \pm 9.4$ & $35.3 \pm 3.7$ & $28.8 \pm 4.0$ & $27.5 \pm 6.5$ \\
\hline
\end{tabular}

${ }_{\text {, }} \mathrm{p}<0.05 ;{ }^{* *}, \mathrm{P}<0.01$; compared with model group; \#,P<0.05, compared with sham-operated

group. 
concentration of dopamineDA. The coefficient was calculated to be 0.9997 . The intraday relative standard deviation (RSD) was $2 \%$, and inter-day RSD was $2.8 \%$. The recovery exercise was determined at low, medium and high concentrations of QC samples, and the values were calculated to be $96.67 \% 106.00 \%$ and $92.50 \%$, respectively.
The influence of Ginkgo Bilboa injection towards the DA level in brain

Based on the established analytical approach, the concentrations of DA in striatum of rat brain from the different sampling point were tested (Fig 2). At the same time, the DA level in brain was determined at the different sampling time, and the results were given in Table 1. The DA level in corpus striatum remained

\section{Figure Legends}

Fig.1 Representative figures for determination of different concentrations of dopamine (DA).

Fig.2 Chromatograms of DA concentration in aCSF from different group. A: sham-operatec group; B: ischemia-reperfusion group; C: nimodipine injection group; D: Ginkgo Biloba injectior group.

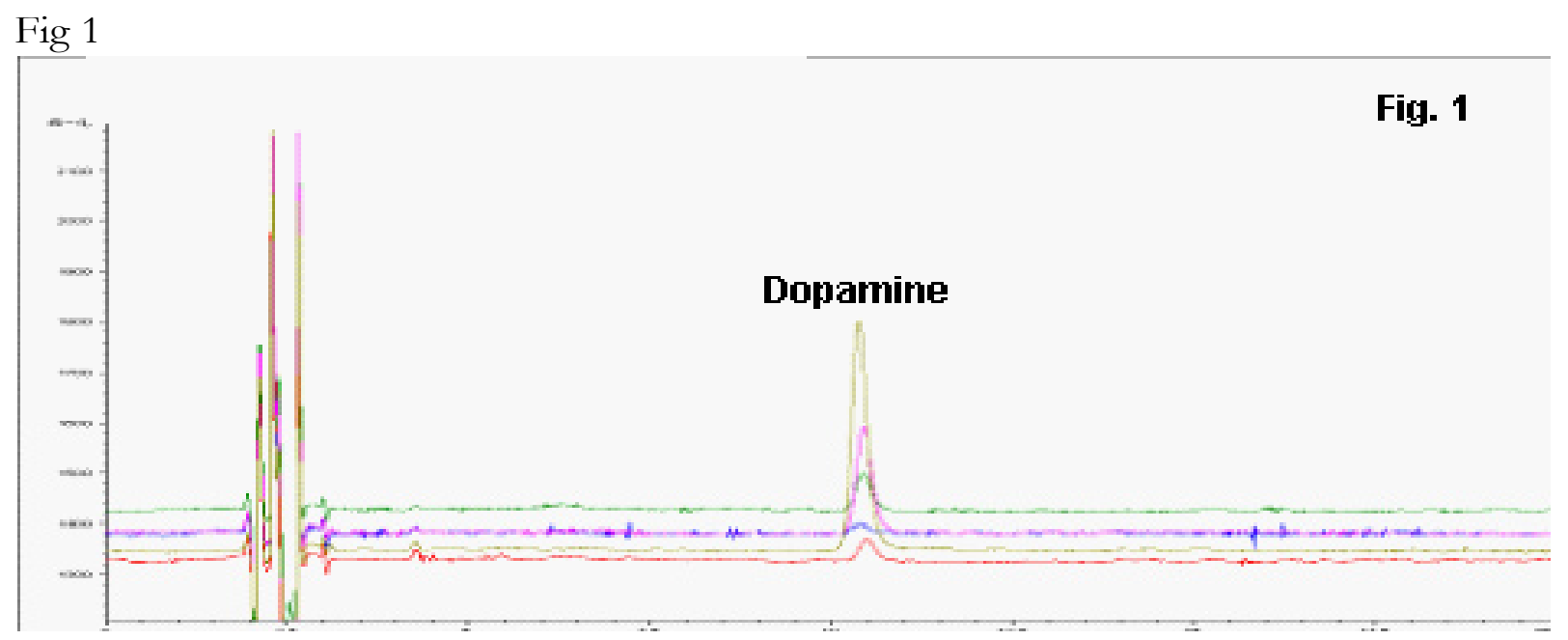

stable between 0 and $60 \mathrm{~min}$ in which the treatment was not given. The i.p. administration of Ginkgo Bilboa injection and nimodipine injection was began at $60 \mathrm{~min}$. After $30 \mathrm{~min}$ administration, the concentration of DA was higher in Ginkgo Bilboa injection group and nimodipine injection group. However, no significant difference was observed between treatment group and model group. At 90 min, ischemia model was constructed, and after 30 min, comparing with sham-operated group, the DA level in ischemia model exhibited significantly higher $(\mathrm{p}<0.01)$. The DA level in Ginkgo Bilboa injection group $(p<0.01)$ and nimodipine injection group $(\mathrm{p}<0.05)$ was much lower than model group. At $120 \mathrm{~min}$, the reperfusion was performed. The DA level in ischemia model group immediately decreased to pre-ischemia DA level. At $180 \mathrm{~min}$, the DA level increased, and then recovered to normal level. Different from the model group, the DA level in Ginkgo Bilboa injection group showed gradually decrease trend, and did not exhibit the recovery phenomena.

\section{Discussion}

Cerebral ischemia-reperfusion (I/R) injury has had been considered as the major pathogenic mechanism of various cerebrovascular diseases 6. For the traditional method, the determination of neurotransmitter needs needed to take brain tissue through decapitating the rats. Additionally, the precipitation of protein was needed after homogenization of brain tissues. This method is was very complicated, and needs requried 
large numbers of experimental animals. Microdialysis is was a relatively novel sampling technique which has had extensively applied in neurotransmitter research, particularly in the determination of monoamine and aminoacid neurotransmitters 7 . In combination with the rapid development of some analytical methods (e.g. liquid chromatography, mass spectrometry, electrochemical detection, etc), themicrodialysis methodology has had been successfully applied in neurochemistry 8 .

In the present study, this technology was employed to investigate the therapeutic role of Ginkgo Bilboa injection towards cerebral ischemia-reperfusion (I/R) injury. Similar with the results reported by $\mathrm{Li}$ et al.9, in the $\mathrm{I} / \mathrm{R}$ injury model, the concentration of DA sharply increased to the peak value after $30 \mathrm{~min}$ ischemia. After the reperfusion, the concentration rapidly decreased to the normal level. After cerebral ischemia, nimodipine injection and Ginkgo Biloba injection significantly prevent the elevation of DA level, indicating that these two injections can efficiently control the metabolic disorders of DA. During reperfusion process, the influence of nimodipine injection and Ginkgo Biloba injection towards DA level exhibited different behaviors. For nimodipine injection group, the DA level continued to increase, which might be due to the short half life (1.1-1.7 h) of nimodipine injection. In Ginkgo Biloba injection group, the DA level gradually decreased, and DA level remained at the baseline level for a longer time, indicating Ginkgo Biloba injection had the more efficient than nimodipine injection.

\section{Conflict of interest}

None of the authors have any conflict of interest.

\section{Conclusion}

The present study indicated the effective treatment of
Ginkgo Biloba injection towards I/R injury through the determination of dopamineDA level alteration using MD-HPLC-ECD method.

\section{References}

1. Markaki I, Franzen I, Talani C, Loizou L, Kostulas N. Long-term survival of ischemic cerebrovascular disease in the acute inflammatory stroke study, a hospital-based cohort described by TOAST and ASCO. Cerebrovasc. Dis. 2013; 35(3):213-219.

2. Pan J, Konstas AA, Bateman B, Ortolano GA, Pile-Spellman J. Reperfusion injury following cerebral ischemia: pathophysiology, MR imaging, and potential therapies. Neuroradiology 2007; 49(2):93-102.

3. Harukuni I, Bhardwaj A. Mechanisms of brain injury after global cerebral ischemia. Neurol. Clin. 2006; 24(1):1-21.

4. Wilson L, Sheehan J, Thorpe M. Three cases of impulse control disorder in Parkinson's disease patients receiving dopamine replacement therapy. Ir. Med. J. 2013; 106(1):24-25.

5. Borta A, Höglinger GU. Dopamine and adult neurogenesis. J. Neurochem. 2007; 100(3):587-595.

6. Takashima S, Itoh M, Oka A. A history of our understanding of cerebral vascular development and pathogenesis of perinatal brain damage over the past 30 years. Semin. Pediatr. Neurol. 2009; 16(4):226-236.

7. Bourne JA. Intracerebral microdialysis: 30 years as a tool for the neuroscientist. Clin. Exp. Pharmacol. Physiol. 2003; 30:16-24.

8. Perry M, Li Q, Kennedy RT. Review of recent advances in analytical techniques for the determination of neurotransmitters. Anal. Chim. Acta. 2009; 653:1-22.

9. Li H, Li C, Yan ZY, Yang J, Chen H. Simultaneous monitoring multiple neurotransmitters and neuromodulators during cerebral ischemia/reperfusion in rats by microdialysis and capillary electrophoresis. J. Neurosci. Methods 2010; 189(2): 162-168. 